\title{
A New Way Study of Embedded PLC Control System Fault Diagnosis
}

\author{
LIU ChangLiang ${ }^{1, a}$ \\ ${ }^{1}$ Guangxi Vocational \& Technical Institute of Industry, Nanning 530001,China \\ aliuchangliang@163.com
}

Keywords: Embedded PLC; Fault Diagnosis; PLC Control

\begin{abstract}
With the rapid development of PLC and its widely used in the field of industrial, life, etc., is bound to bring high performance requirements of PLC. Reliability for measure of PLC performance indicators, and PLC application in most occasions must meet the requirements. How to improve the reliability of the PLC at the same time, and shall not affect the PLC program execution speed, is a question of this article needs to discuss. This article obtains from the PLC control principle and realization method, on the basis of the theoretical and experimental research, combining soft PLC technology and embedded system, this paper proposes a new method of fault diagnosis, was designed and implemented the embedded PLC system with soft fault diagnosis ability, improve the system reliability.
\end{abstract}

\section{Introduction}

With the rapid development of modern industry and science and technology, the technology is increasingly competitive, industrial enterprises one of the key factors of success is the quality of the product and manufacturing process control, especially the development of computer technology, the structure of the equipment is more and more complex, more and more is also high degree of automation, not only related to each other between different parts of the same equipment, tight coupling, but also between different devices there are closely linked, in the running process to form a whole [1-2]. Therefore, a fault may cause a series of chain reaction, cause the entire equipment and even the whole process cannot run normally, light person caused production downtime, the person that weigh can have serious or even catastrophic casualties. At present, the equipment fault diagnosis has become one of hot topics in the study of modern industrial production.

Programmable logic controller (PLC) play an extremely important role in the industrial field. Now the growing demand for industrial control of PLC with higher performance requirements, much attention has been paid to the reliability requirements [3]. Improve the reliability of PLC can generally be from two aspects of hardware and software, hardware mainly through redundancy technology enhance the tolerance of the system, in the event of a failure to ensure that the system is still maintain normal work, but as a direct result of the rising cost of development, also increased the complexity of the development process; Software mainly depend on the PLC since the detection ability or USES the fault diagnosis technology, and to join in the traditional PLC system fault diagnosis function, will expand the scope of application, and extend the scan cycle, the PLC to timely response requirements of the real-time control is difficult to meet.

\section{The overall structure of the embedded PLC}

Embedded PLC system structure diagram as shown in Figure 1, the development of system of computer soft in the PLC diagram, mainly to complete the ladder diagram programming, ladder diagram instruction code table, and other functions, the realization of [4] is responsible for transporting the embedded microprocessor users and PLC control program. Embedded microprocessor PLC as the control core, compile and execute the PLC user program, the FPGA circuit is used to extend the I / O, I / O point flexible. Connect the PC through the CAN bus interface card and CAN bus, PC embedded microcontroller processor and CAN bus communication, embedded microprocessor and I / O expansion module communication, I / O and extended PLC 
system[5]. Embedded PLC system will develop and run a separate operation, the use of computer users of ladder diagram program development, application and development process of the computer (PC) design platform, the development of soft PLC system in the embedded system, execution of the user program, through the CAN bus communication and control system modules of computer and soft PLC distributed control network.

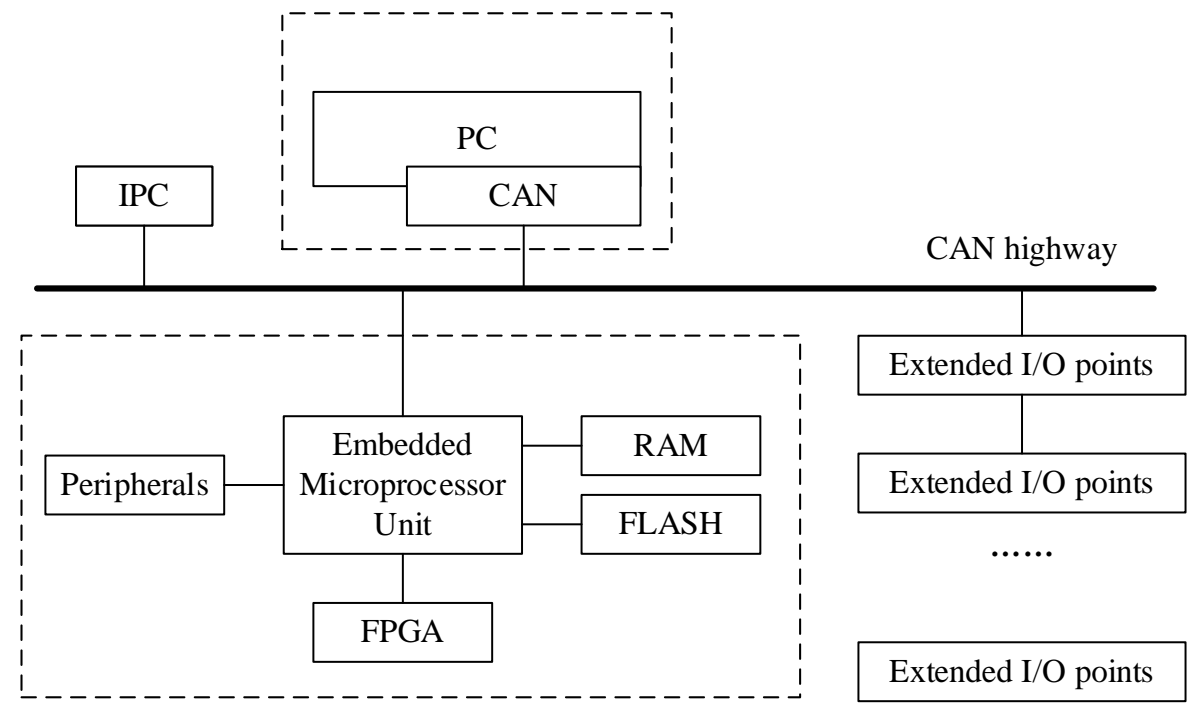

Figure 1. The embedded PLC system structure

Embedded PLC system can be divided into two parts: the development system and running system. Development system running on the PC platform, provides editing, compilation, debugging, simulation functions, and other functions, operating system running on the embedded hardware platform, responsible for the target code analysis company. Object code is the bridge of development system and running system, development system connection will write good PLC application program compiler to generate the corresponding configuration file connection, and then download the configuration file system operation, the operating system will parse the contents of the configuration file, using PLC realize a complete specific logic control.

\section{The embedded PLC hardware design}

According to the demand analysis of the embedded PLC, embedded PLC modular design: the main control module, data will be only responsible for the operation and treatment. The host, the host through a variety of bus and Ethernet, and other communication module; field data collection, data processing is realized between the output of the PLC input and output module, I / O ports of CPU and photoelectric isolation and prevent interference, CPU also plays a role in protection. The use of high performance embedded micro controller host CPU, make full use of its internal integration of resources, structure + interface, simplified PLC CPU supplemented with SDRAM, peripherals, FLASH and other basic systems need to be equipped with the Ethernet controller and bus interface to communicate with other devices, the hardware block diagram as shown in figure 2.

It can be seen from the system, the key part of LPC2292 hardware structure of PLC control system of the main controller, it can use the integrated analog interface, switch interface, RS232 interface, SPI bus acquisition of field data, can also through the instrument and can bus industrial control field or other equipment connected by means of real-time data collection, embedded Web industrial field the remote monitoring platform, user control command PLC sent through the Internet, to realize remote monitoring of industrial field. 


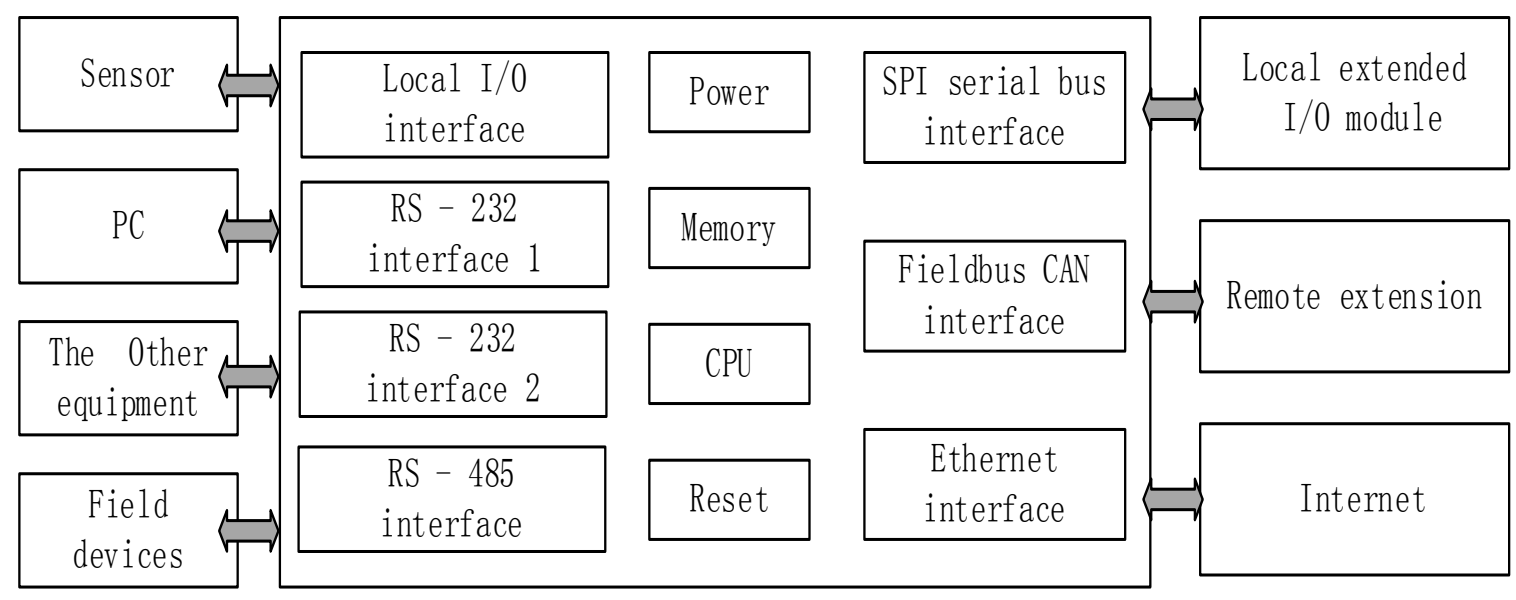

Figure 2. The Embedded PLC system hardware structure

\section{Overall design of embedded PLC control system fault diagnosis}

With functions of fault diagnosis of embedded PLC control system is designed to improve the traditional PLC inefficiency of the single task, on the basis of embedded PLC, will be part of the fault diagnosis system, data acquisition part as a low priority tasks, embedded in the original PLC control system. Then through a serial port transmission control in the process of soft fault characteristic value, switch and analog transmission to the upper machine (PC) diagnosis system, using state machine model to analyze the current state of the control system, and provide troubleshooting tips for the user. Eventually can realize the collection online, offline diagnosis, make full use of the resources of the PLC control system, and improve the reliability of PLC control system. This system only store data acquisition part of the reason is that the next bit machine considering embedded system memory space is limited, if put the complete diagnosis system can increase the burden of system, therefore, choose the scheme, put forward the system overall framework is shown in figure 3.

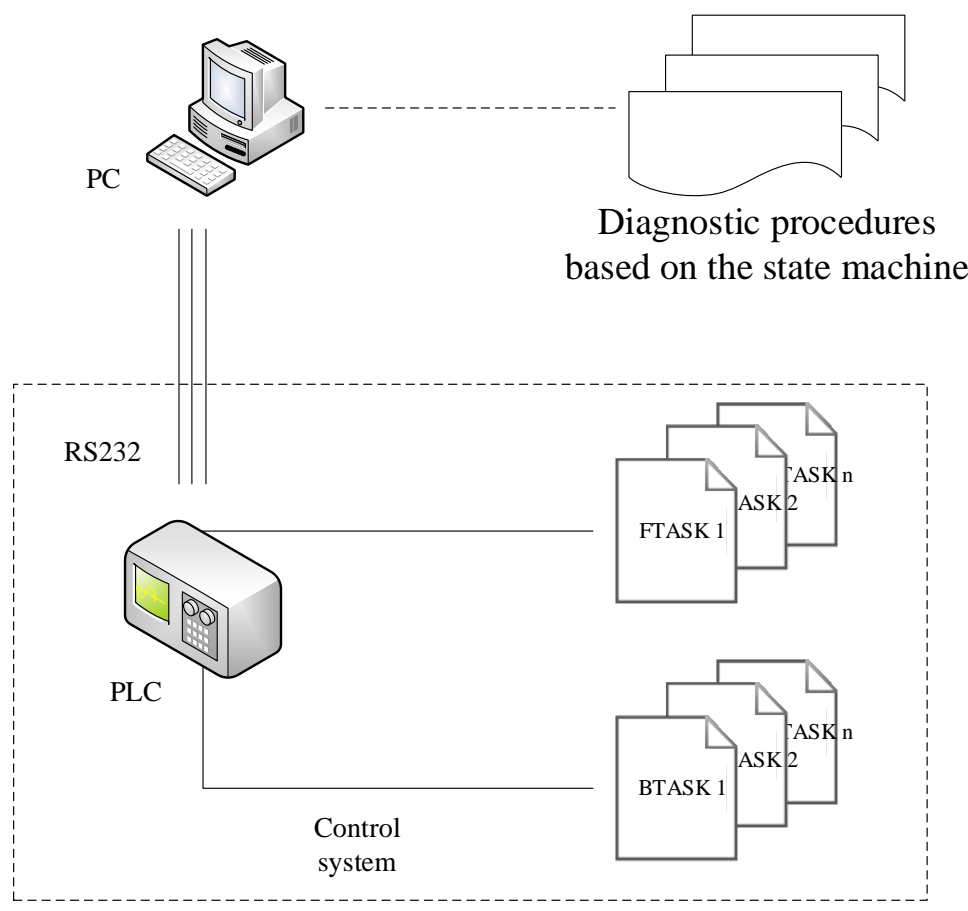

Figure 3. Overall structure of embedded PLC control system fault diagnosis

Equipment control. Equipment control function is the main function of PLC, implement the user program to realize sequence control with the analysis of the charged equipment function. The 
system need to make sure that after adding the diagnosis function, the control function of the original is not affected, and try to maintain the existing control performance.

Online data collection. Data acquisition task is realized in the embedded PLC control system, the source for fault diagnosis system after diagnosis, is the basis of the diagnosis system. Online data acquisition task only simple data processing ability, the implementation code is relatively short, so won't occupy too large memory, ensure that the performance of the control system of the original.

Upper machine fault diagnosis. In the embedded PLC control system diagnostic data were collected through communication transmission to the PC after diagnosis system, which can be used in the construction of a state machine model and normal formal diagnosis process of data analysis. Upper machine fault diagnosis system is the realization of the function of diagnosis platform, the work includes the early stage of the state machine for the training process, the use of model specification fault diagnosis model and data process, and perfecting process of specification model.

The whole system is divided into upper machine diagnosis system and control system of two parts. Control system implementation of accused of equipment control and diagnostic data collection and transmission, and realize the two big tasks run in parallel; Diagnosis system receives the transmission to the switch quantity or analog data, such as diagnosis combined with early training of state machine model specifications complete fault detection and diagnosis process.

\section{Conclusion}

PLC play an extremely important role in the industrial field. Now the growing demand for industrial control of PLC with higher performance requirements, much attention has been paid to the reliability requirements. Scientific research work of this paper for the research of high reliability PLC provides a new method of heuristic exploration and attempt, the calculation of embedded PLC task scheduling mechanism. Considering the non-lethal soft fault on system operation, its diagnosis part as a low-level thread can be embedded into the original control system, to realize the harmonious operation of control tasks and diagnostic tasks, improve the performance of PLC and the system reliability.

\section{Reference}

[1] R. Bayindir, Y. Cetinceviz: ISA transactions, Vol.5 (2013) No.2, p. 321.

[2] Z. Aydogmus, O. Aydogmus: Education, Vol.2 5(2009) No.1, p. 126.

[3] M. Kano, M. Ogawa: Journal of Process Control, Vol.20 (2012) No.9, p.969.

[4] G. Valencia-Palomo, J.A. Rossiter: ISA transactions, Vol.36 (2008) No.1, p. 92.

[5] J.C.B. Gonzaga, L.A.C. Meleiro, and C. Kiang: Computers \& Chemical Engineering, Vol.33 (2009) No.1, p. 43. 